\title{
Long-range Tamm surface plasmons supported by graphene-dielectric metamaterials
}

Hodjat Hajian, Humeyra Caglayan, and Ekmel Ozbay

Citation: Journal of Applied Physics 121, 033101 (2017); doi: 10.1063/1.4973900

View online: https://doi.org/10.1063/1.4973900

View Table of Contents: http://aip.scitation.org/toc/jap/121/3

Published by the American Institute of Physics

\section{Articles you may be interested in}

Asymmetric transmission of linearly polarized waves in terahertz chiral metamaterials Journal of Applied Physics 121, 033103 (2017); 10.1063/1.4974477

Photonic crystal properties of self-assembled Archimedean tilings

Journal of Applied Physics 121, 023101 (2017); 10.1063/1.4973472

Terahertz plasmonic rectification in a spatially periodic graphene

Applied Physics Letters 110, 061106 (2017); 10.1063/1.4975829

Telecom wavelength emitting single quantum dots coupled to InP-based photonic crystal microcavities Applied Physics Letters 110, 031101 (2017); 10.1063/1.4974207

Microfluidic metamaterial sensor: Selective trapping and remote sensing of microparticles Journal of Applied Physics 121, 023102 (2017); 10.1063/1.4973492

Highly tunable bistability using an external magnetic field in photonic crystals containing graphene and magnetooptical layers

Journal of Applied Physics 121, 023105 (2017); 10.1063/1.4973897

\section{PHYSICS TODAY}

MANAGER'S GUIDE

WHITEPAPERS

\section{READ NOW}

PRESENTED BY

Accelerate R\&D with

Multiphysics Simulation
COMSOL 


\title{
Long-range Tamm surface plasmons supported by graphene-dielectric metamaterials
}

\author{
Hodjat Hajian, ${ }^{1, a)}$ Humeyra Caglayan, ${ }^{1}$ and Ekmel Ozbay ${ }^{1,2,3}$ \\ ${ }^{1}$ Nanotechnology Research Center, Bilkent University, 06800 Ankara, Turkey \\ ${ }^{2}$ Department of Physics, Bilkent University, 06800 Ankara, Turkey \\ ${ }^{3}$ Department of Electrical and Electronics Engineering, Bilkent University, 06800 Ankara, Turkey
}

(Received 24 October 2016; accepted 28 December 2016; published online 17 January 2017)

\begin{abstract}
Considering the Ohmic losses of graphene in the calculations and by obtaining exact dispersion relations of the modes, we theoretically study propagation and localization characteristics of Tamm surface plasmons supported by terminated graphene metamaterials. The metamaterials are composed of alternating layers of graphene and dielectric with subwavelength periods. We also examine the Tamm modes within the framework of long-wavelength approximation. It is shown that, in case the Ohmic losses of the graphene layers are taken into account, surface plasmons are not supported in a long-wavelength region, in which the graphene-dielectric multilayer structure behaves as a hyperbolic metamaterial. We prove that, when the metamaterial is truncated with air, by choosing sufficiently thick but still subwavelength dielectric layers, i.e., $d=300 \mathrm{~nm}$, these surface waves will have a moderate propagation (localization) length that is comparable with those of a single layer of graphene. On the other hand, in case a miniaturized graphene metamaterial $(10<d(\mathrm{~nm})<100)$ is truncated by a thick cap layer $\left(d_{c a p}=5 d\right)$ with $\varepsilon_{c a p}>\varepsilon_{\text {dielectric }}$, it is possible to considerably improve the propagation and localization characteristics of the Tamm modes supported by the system within the $5.5-50 \mathrm{THz}$ range of frequency, as compared to a single layer of graphene. Published by AIP Publishing. [http://dx.doi.org/10.1063/1.4973900]
\end{abstract}

\section{INTRODUCTION}

In analogy to the electronic Tamm states supported at the surface of semi-infinite superlattices, ${ }^{1}$ some optical systems are also capable of supporting electromagnetic version of these modes, which are called $\mathrm{Bloch}^{2}$ or $\mathrm{Tamm}^{3-8}$ surface waves. They have attracted widespread attention in recent years due to their applications in guiding, sensing, ${ }^{5,6}$ and lasers. ${ }^{8}$ Similar to surface plasmons (SPs) guided at conductor-dielectric interfaces for transverse magnetic polarization (TM), ${ }^{9}$ Tamm surface modes propagate at an interface between nonhomogeneous and homogeneous/nonhomogeneous media, evanescently confined in the perpendicular direction for TM polarization and/or transverse electric (TE) polarization. ${ }^{10}$ As a couple of examples, an interface separating a photonic crystal (PC) and homogeneous medium, ${ }^{2,5-8,10-12}$ an interface between two $\mathrm{PCs}^{8,9,13}$ and an interface between a semiinfinite PC and left-handed metamaterials ${ }^{14}$ can support these modes. In addition to the mentioned systems, metaldielectric multilayer structures can also support Tamm surface waves. ${ }^{15,16}$ Since the dispersion of these modes resembles that of SPs of a metal-dielectric interface, they can be called Tamm surface plasmons (Tamm SPs). It is noteworthy that in case the thickness of the layers in metal-dielectric structures is subwavelength, they can be effectively considered as a uniaxially anisotropic hyperbolic metamaterial (HMM) with the hyperbolic form of dispersion relation. ${ }^{17}$ HMMs promise a variety of potential

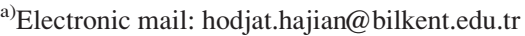

applications such as engineering of spontaneous emission, broadband Purcell effect, subwavelength imaging, negative refraction, and waveguides. ${ }^{18,19}$

After the rise of graphene, ${ }^{20}$ considerable scientific attention has been paid to this material due to its incredible potential applications in optoelectronics ${ }^{21}$ and plasmonics. $^{22}$ The surface conductivity of graphene $\left(\sigma_{g}\right)$, which is a function of frequency (f), temperature $(\mathrm{T})$, and electron relaxation time $(\tau)$, can also be effectively modulated via tuning of chemical potential $(\mu)$ through chemical doping or electrostatic/magnetostatic gating. ${ }^{20}$ For $\operatorname{Im}\left(\sigma_{g}\right)>0$, graphene behaves like a very thin metal layer capable of supporting transverse magnetic (TM) surface plasmons (SPs). ${ }^{23}$ Tunability of its plasmon resonance through the variation of $\mu$ together with a relatively large propagation length (PL) and a small localization scale of SPs in the infrared (IR) and terahertz $(\mathrm{THz})$ ranges are the key advantages of graphene SPs over those supported by noble metals like silver and gold. ${ }^{23}$ Therefore, inspired by the metal-dielectric HMMs, graphene-dielectric hyperbolic metamaterials have been theoretically predicted in $\mathrm{THz}$ and far-infrared (IR) frequency regions ${ }^{24-26}$ and experimentally proved at mid-IR. ${ }^{27}$ It has been suggested that these systems can also be used for the enhancement of spontaneous emission, ${ }^{25,26}$ waveguides, ${ }^{28,29}$ tunable broadband hyperlense, ${ }^{30}$ negative refraction, ${ }^{31}$ and perfect absorption. ${ }^{32}$ Within the framework of nonlocal effective medium theory (EMT), ${ }^{25}$ it has been reported that graphene-dielectric metamaterials (GMMs) can support additional waves known as polariton modes. ${ }^{33}$ Moreover, it has been demonstrated that graphene metamaterials are capable of supporting different types of modes such as 
Tamm SPs ${ }^{34}$ surface Bloch waves, ${ }^{35}$ long-range $\mathrm{SPs},{ }^{28}$ short-range SPs and bulk polaritons, called as high-k propagating modes. ${ }^{36}$ It is noteworthy that in case the thickness of the dielectric layers is not subwavelength, multilayer graphene-dielectric systems may also support the mentioned modes. 37,38

In this paper, considering the Ohmic losses of graphene layers, we examine the propagation and localization characteristics of the Tamm SPs supported by realistic graphenedielectric metamaterials. Moreover, we suggest optimal designs of these systems, which are capable of supporting long-range Tamm SPs within 5.5-50 THz. To the best of our knowledge, the results presented here have not been reported elsewhere.

\section{MATH AND EQUATIONS}

\section{A. Exact dispersion relations of Tamm surface plasmons}

As schematically shown in Fig. 1(a), the graphene metamaterial is composed of a periodic arrangement of graphene layers with surface conductivity $\sigma_{g}$ and period $d$, embedded in a background with the dielectric constant $\varepsilon$. This system can also be capped with a medium of thickness $d_{c}$ and dielectric constant $\varepsilon_{c}$ (see Fig. 1(b)). Letting the wave vector lie in the $\mathrm{xz}$ plane, it is well known that the TM-polarized dispersion relation of this system can be given by ${ }^{21,22,34,35}$

$$
\cos \left(K_{B} d\right)=\cosh (q d)-\frac{\alpha q}{2 \varepsilon} \sinh (q d) .
$$

Here, $K_{B}$ is the Bloch wave number, $q=\sqrt{\beta^{2}-\varepsilon \beta_{0}^{2}}$, $\beta=k_{x}, \beta_{0}=\omega / c, \alpha=\sigma_{g} / i \omega \epsilon_{0}$, and we use Falkovski's formalism to include both inter- and intra-band contributions in $\sigma_{g}{ }^{21}$ First, we obtain exact dispersion relation of Tamm SPs in the absence of the cap layer. For a graphene metamaterial truncated at $z=0$ with a semi-infinite homogeneous medium of $\varepsilon_{s}, H_{y}$ may be considered as

$$
H_{y}(z)=\left\{\begin{array}{ll}
A[\sinh (q z)+\gamma \cosh (q z)] & z \geq 0 \\
B \exp \left(q_{s} z\right), & z<0
\end{array}\right\},
$$

where $q_{s}=\sqrt{\beta^{2}-\varepsilon_{s} \beta_{0}^{2}}$. By applying the following standard boundary conditions for TM polarization ${ }^{21,34,37}$

$$
\left.E_{x}\right|_{z=0^{+}}=\left.E_{x}\right|_{z=0^{-}},
$$

and

$$
\left.\frac{\varepsilon_{s}}{q_{s}^{2}} \frac{d E_{x}}{d z}\right|_{z=0^{-}}-\left.\frac{\varepsilon}{q^{2}} \frac{d E_{x}}{d z}\right|_{z=0^{+}}=\left.\alpha E_{x}\right|_{z=0}
$$

on Eq. (2), we arrive at the below dispersion relation for the Tamm SPs, which is in agreement with that reported by Ref. 31

$$
\frac{\varepsilon_{s}}{q_{s}}-\frac{\varepsilon \gamma}{q}=\alpha
$$

Here, $\gamma$ can be obtained by applying the boundary conditions together with the Bloch periodic ones on the first period of the metamaterial as

$$
\gamma=\frac{e^{i K_{B} d}-\cosh (q d)}{\sinh (q d)} .
$$

It is noteworthy that this method of obtaining $\gamma$ has been called the direct-matching procedure ${ }^{39}$ and can be applied in any periodic layered system. ${ }^{40}$ In case the graphene metamaterial is truncated with a cap layer of width $d_{c}$ and dielectric constant of $\varepsilon_{c}$ (see Fig. 1(b)), the exact dispersion relation of the Tamm SPs can be derived as

$$
\tanh \left(q_{c} d_{c}\right)=\frac{\eta+\eta_{s}}{1+\eta \eta_{s}}
$$

where $q_{c}=\sqrt{\beta^{2}-\varepsilon_{c} \beta_{0}^{2}}, \eta=q_{c}(\varepsilon \gamma / q+\alpha) / \varepsilon_{c}$ and $\eta_{s}=-q_{c} \varepsilon_{s} /$ $q_{s} \varepsilon_{c}$.

\section{B. Long-wavelength approximation of Tamm surface plasmons}

As reported previously, ${ }^{24}$ in the long-wavelength limit, $q d \ll 1$, Eq. (1) can be reduced to a hyperbolic dispersion relation, $K_{B}^{2} / \varepsilon_{x}+\beta^{2} / \varepsilon_{z}=\beta_{0}^{2}$ where, in the local EMT formalism, $\varepsilon_{z}$ and $\varepsilon_{x}$ can be presented as $\varepsilon$ and $\varepsilon_{z}-\alpha / d$, respectively. Consequently, the dielectric response of the multilayer graphene metamaterial can be effectively described as that of a uniaxially anisotropic medium with a dielectric tensor of

$$
\varepsilon_{e f f}=\left[\begin{array}{ccc}
\varepsilon_{x} & 0 & 0 \\
0 & \varepsilon_{y} & 0 \\
0 & 0 & \varepsilon_{z}
\end{array}\right],
$$
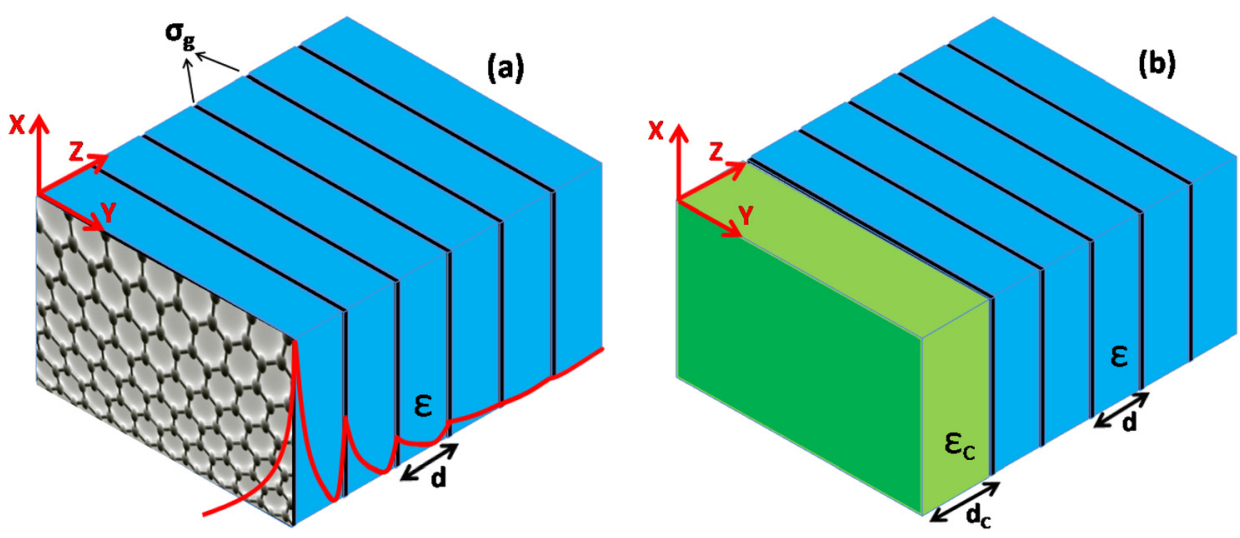

FIG. 1. Panels (a) and (b) show the schematics of the graphene metamaterial without and with the presence of a cap layer, respectively. The dielectric layers separating graphene sheets in the metamaterial have a subwavelength thickness of $d$ with the dielectric constant of $\varepsilon$. The dielectric constant and thickness of the cap layer are $\varepsilon_{c}$ and $d_{c}$. Both structures are truncated with semiinfinite homogeneous medium $(z<0)$ of $\varepsilon_{s}$ at $z=0$. The red curve in panel (a) illustrates the $\left|E_{x}\right|$ profile of a typical Tamm surface plasmon supported by the graphene-dielectric metamaterial. 
where $\varepsilon_{x}=\varepsilon_{y}$. In this approximation, $\gamma$ can be re-written as

$$
\gamma=\frac{-\alpha q}{2 \varepsilon_{z}}+\sqrt{\frac{\varepsilon_{x}}{\varepsilon_{z}}} .
$$

And, therefore, the dispersion relation of the Tamm SPs presented in Eqs. (4) and (6) reduces to

$$
\beta=2\left(\varepsilon_{s}-\sqrt{\varepsilon_{x} \varepsilon_{z}}\right) / \alpha
$$

and

$$
\beta=-B^{\prime} \pm \sqrt{\Delta} / 2 A^{\prime}
$$

respectively, where $A^{\prime}=\varepsilon_{s} d_{c} \alpha / 2 \varepsilon_{c}, B^{\prime}=\alpha / 2-\varepsilon_{c} d_{c}+\varepsilon_{s} d_{c}$ $\sqrt{\varepsilon_{x} \varepsilon_{z}} / \varepsilon_{c}, C^{\prime}=\sqrt{\varepsilon_{x} \varepsilon_{z}}-\varepsilon_{s}$, and $\Delta=\sqrt{B^{\prime 2}-4 A^{\prime} C^{\prime}}$. It is noteworthy that at $q d \ll 1$ condition, for which the effectivemedium approximation is valid, neither the phase nor the amplitude of a propagating wave does vary significantly across the thickness of any layer in the metamaterial. This condition is also called as a "subwavelength condition" for HMMs. ${ }^{41}$ It should be noticed that in conventional media the subwavelength condition can simply be $d / \lambda \ll 1$ due to the elliptical dispersion relation, which restricts the range of possible $\beta$. While in the hyperbolic metamaterials, the "subwavelength condition" breaks down for some large $\beta$ no matter how thin the layers are.

\section{RESULTS AND DISCUSSION}

In this section, we investigate Tamm SPs supported by the graphene metamaterials presented in Fig. 1. In order to obtain a concise insight into the effect of the Ohmic losses of graphene sheets on the modes characteristics, we first consider the lossless case. Then, we revise the results by including the losses in the calculations. It should be noted that we choose the following material parameters in the calculations of the optical conductivity of graphene: $\tau=1.35 \times 10^{-13} \mathrm{~s}$, $T=300$, and $\mu=0.2 \mathrm{eV}$. Moreover, the dielectric constant of the dielectric layers $(\varepsilon)$ in the GMMs and that of the cap layer $\left(\varepsilon_{s}\right)$ are taken as 4 (Refs. 25 and 40) and $10,{ }^{34}$ respectively.

\section{A. Lossless case}

In case we neglect the losses, the plot of Eq. (1) leads to the band structure of the metamaterial that can be divided into allowed and forbidden regions. For the allowed regions (turquoise regions in Fig. 2), $K_{B}$ is real, hence the modes placed in this region can propagate inside the metamaterial. While, for the forbidden bands or bandgaps (white regions in Fig. 2), the Bloch wavenumber becomes imaginary and, consequently, the modes supported in this region are evanescent with strong localization. Panels (a), (b), (c), and (d) are plotted for different periods; i.e., for $d=10 \mathrm{~nm}, d=50 \mathrm{~nm}$, $d=100 \mathrm{~nm}$, and $d=300 \mathrm{~nm}$, respectively. In these panels, black dashed lines show the dispersion of a single layer of graphene (SLG) that is bounded with air and dielectric material with $\varepsilon=4$. As shown in panel (a), when the spacing between adjacent graphene layers is $10 \mathrm{~nm}$, the allowed modes appear in a wide range of frequency for the specific values of the parallel wavevector $\beta$. When the period of the metamaterial is taken in such a way that the surface plasmon modes supported at each layer are decoupled, the allowed band shrinks to the dashed-dotted curve in panel (a), which corresponds to the dispersion of a SLG bounded with the dielectric material. Using Eqs. (4) and (6), the exact dispersion curves of Tamm SPs supported by the GMM without and with the presence of the cap layer are plotted in dashed-
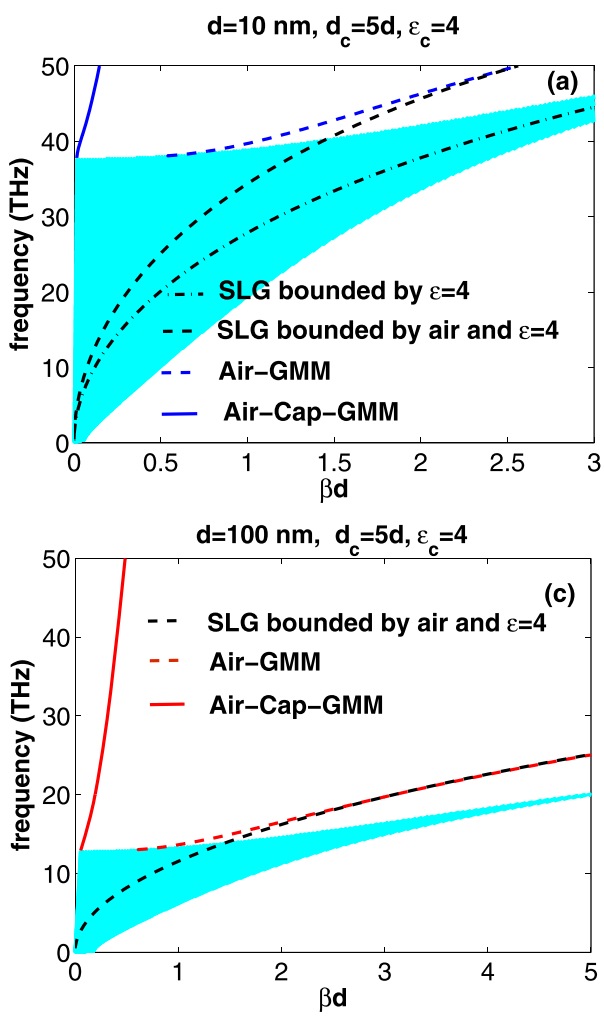
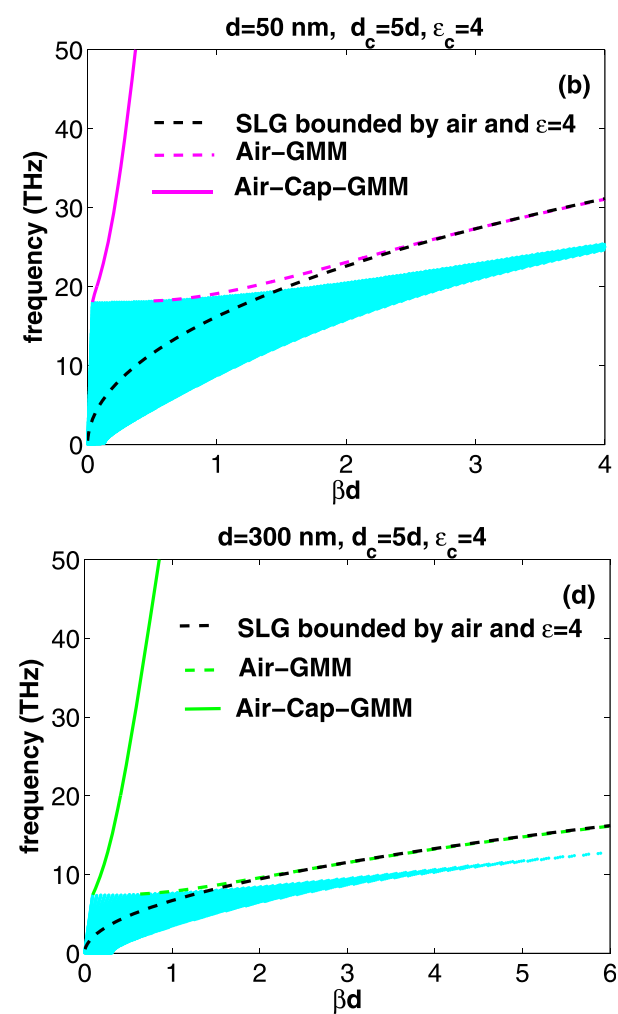

FIG. 2. Turquoise and white regions in these panels show the allowed and forbidden bands in the band structure of the graphene metamaterials with $d=10 \mathrm{~nm}$ (panel (a)), $d=50 \mathrm{~nm}$ (panel (b)), $\quad d=100 \mathrm{~nm} \quad$ (panel (c)), and $d=300 \mathrm{~nm} \quad$ (panel (d)). The black dashed curves show the dispersion of SPs supported by a single layer of graphene bounded by air and a dielectric of $\varepsilon=4$. Besides, the solid and dashed colored curves in these panels represent the Tamm surface plasmons of the GMMs with and without the cap layer, respectively. In panel (a), the black dashed-dotted curve shows the dispersion of SPs of a single layer of graphene, which is symmetrically bounded by the dielectric material of $\varepsilon=4$. 
and solid-blue curves in panel (a), respectively. For these modes $\operatorname{Re}\left(K_{B}\right)=0$ and, therefore, they can also be called pure surface plasmons ${ }^{37}$ when $\operatorname{Im}\left(K_{B}\right)>0$. It is important to point out the effect of the dielectric constant of the cap layer on the modes; when $\varepsilon_{c}<\varepsilon$, the modes with $\operatorname{Im}\left(K_{B}\right)>0$ and similar trend of the air-truncated Tamm SPs can be supported by the system for large values of $\beta d(\beta d>0.5)$. While as shown by solid curves in panel (a) and the other panels, for $\varepsilon_{c}>=\varepsilon$, modes with very small values of the wavenumber $(\beta d \ll 1)$ appear in the bandgap. Moreover, our calculations show that when we use a doped semiconductor as the cap layer of the metamaterial, ${ }^{34}$ for $\left|\varepsilon_{C}\right|>=\varepsilon$, pure Tamm SPs with $\beta d \ll 1$ can also be supported by the GMM. As it is seen from panels (b), (c), and (d), by increasing the period of the GMM to $300 \mathrm{~nm}$ : (i) the air-truncated modes supported by the system shifted to lower frequencies. (ii) The Tamm SPs in the presence of the cap layer appear in wider ranges of frequency. (iii) The allowed bands shrink to the narrower regions. More importantly, the above-mentioned physical interpretations we presented for panels (a) are also valid for these panels.

As mentioned above, in case the graphene metamaterial is capped with a dielectric material with $\left|\varepsilon_{C}\right|>=\varepsilon$, Tamm SPs within $\beta d \ll 1$ region can be supported by the system. In this case, long-wavelength approximation $(q d \ll 1)$ may be applied on the exact solutions and we can use Eq. (9b) to investigate the Tamm modes. It has to be pointed out that since the air-truncated graphene metamaterial cannot support Tamm SPs within $\beta d \ll 1$ region, Eq. (9a) is not applicable in this case. Since the graphene metamaterial is capable of having a hyperbolic optical response, it would be constructive to determine the frequency region for which this system acts as a hyperbolic metamaterial. Panel (a) of Fig. 3, in a local effective medium approximation, shows frequency dependency of the " $\mathrm{x}$ " component of the effective permittivity of the GMM. As it is seen from this figure, the graphenedielectric system with a subwavelength period may act as a hyperbolic metamaterial as far as $q d \ll 1$ and we operate at frequencies below $37.7 \mathrm{THz}$ (for $d=10 \mathrm{~nm}$ ) and $18.2 \mathrm{THz}$ (for $d=50 \mathrm{~nm}$ ). Notice that since $\varepsilon_{z}=4$, while operating below these frequencies, the GMM can act as a hyperbolic metamaterial of type II. In support of this point, panel (b) of Fig. 3 illustrates the hyperbolic dispersions of the GMMs at $f=10 \mathrm{THz}$ for two different subwavelength periods. Now, we are in a position to compare the approximate answers of Eq. (9b) to the exact ones of Eq. (6). Fig. 3(c) represents the exact dispersion of Tamm SPs (solid curve) together with the approximate ones (dotted and dashed curves) for $d=10 \mathrm{~nm}$. This figure very clearly illustrates that, first, for $\beta d<0.1$ there is a fair agreement between the exact and approximate answers. And, second, the Tamm SPs are not supported in the frequency region in which the GMM acts as a hyperbolic metamaterial. The same results can be concluded from Fig. 3(d), but the only additional point should be noticed is the considerable difference between the nonretarded answers and the exact ones for $d=50 \mathrm{~nm}$. In another word, for large periods $(d>10 \mathrm{~nm})$, applying a longwavelength limit together with the nonretarded approximation may not be a good tool to examine Tamm SPs in the loss-less case.

\section{B. Lossy case}

So far, we have completely neglected the effect of the Ohmic losses, coming from $\operatorname{Re}\left(\sigma_{g}\right)$, in our analysis. In the case $\operatorname{Re}\left(\sigma_{g}\right)$ is included in the calculations, the parallel
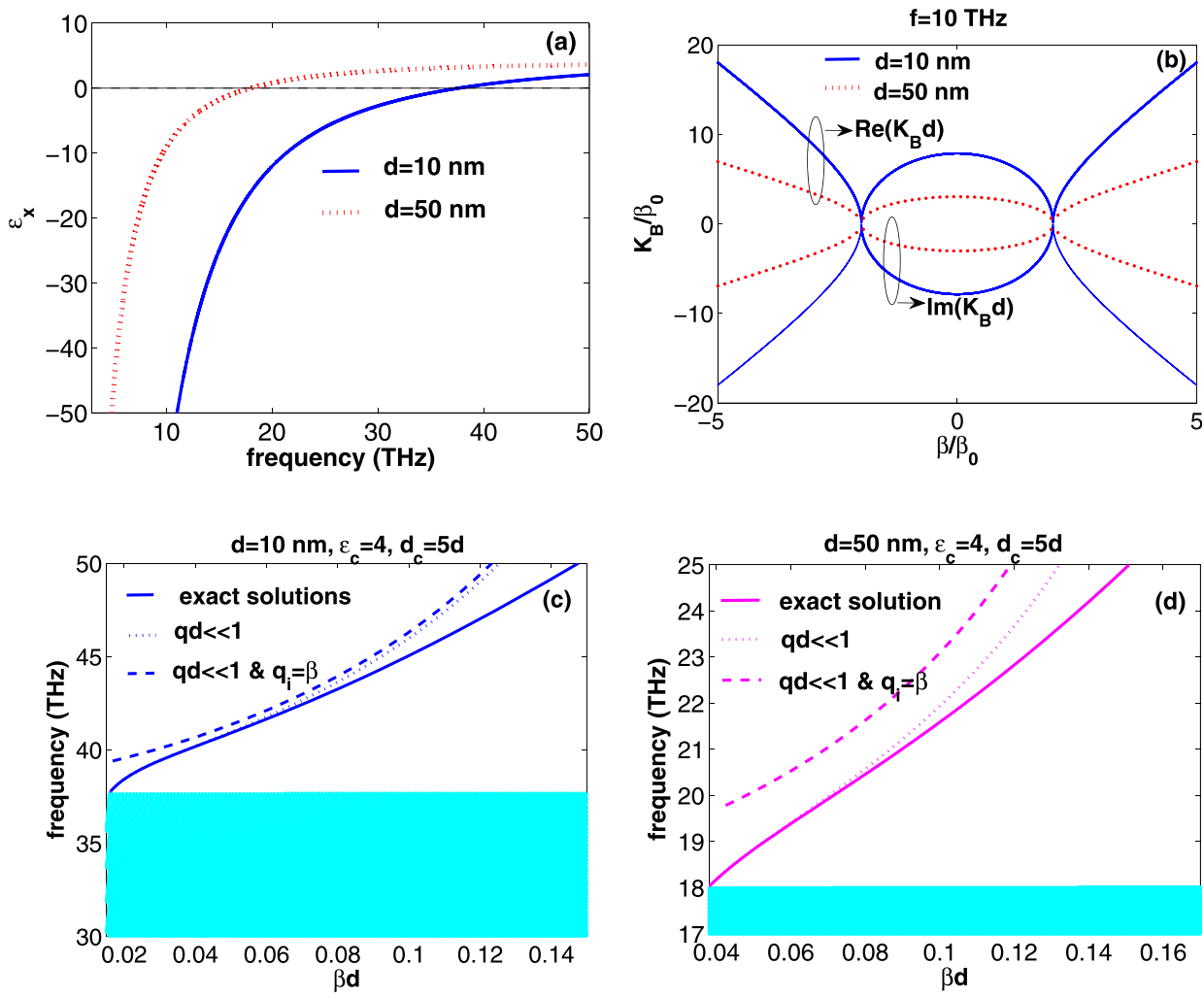

FIG. 3. Panel (a) shows dispersion of the " $x$ " component of the effective permittivity of graphene metamaterial for $d=10 \mathrm{~nm}$ (solid curve) and $d=50 \mathrm{~nm}$ (dotted curve), where $\varepsilon_{z}=\varepsilon=4$. Panel (b) represents the hyperbolic dispersion of the GMMs at $10 \mathrm{THz}$. Dispersion of Tamm SPs of the GMM with $d=10 \mathrm{~nm}$ in the presence of a cap layer with $\varepsilon_{C}=4$ and $d_{C}=5 d$ is represented in panel (c) with solid (exact answers), dotted (long-wavelength approximation), and dashed (longwavelength approximation together with nonretarded one) curves. Panel (d) is similar to panel (c), but for $d=50 \mathrm{~nm}$. 
component of the wavenumber takes complex values, i.e., $\beta=\beta^{\prime}+i \beta^{\prime \prime}$, and consequently the Bloch wavenumber would be complex within the whole $\omega-\beta$ region. In this situation, applying $\left|\cos \left(K_{B} d\right)\right|<1$ condition on Eq. (1) and classifying the band diagram to the allowed and forbidden regions is not acceptable. Therefore, instead of illustrating $\omega-\beta^{\prime}$ dispersions (excluding Fig. 6(a)), we investigate characteristics of Tamm SPs by calculating the normalized propagation length $\left(P L / \lambda_{S P}\right)$ and localization length $(L L)$ of the modes, where $P L=1 / 2 \beta^{\prime \prime}, \lambda_{S P}=2 \pi / \beta^{\prime}$ and $L L=1 /$ $|R e(q)|$. Panels (a) and (b) of Fig. 4 show the normalized PL and LL of Tamm SPs of the air-truncated GMM for different periods. The propagation and localization lengths of the SPs supported by a single layer of graphene are also represented in these figures by black-dashed curves. Besides, in the numerical solutions of the dispersion relations, continuous changes in $\omega$ and $\beta$ are considered. Notice that there is an agreement between the frequency regions of the supported Tamm SPs in Figs. 4(a) and 4(b) with those shown in Fig. 2. We also examined $\omega-\beta^{\prime}$ dispersions for these modes (the corresponding plot is not presented here); similar to the mode dispersions illustrated in Fig. 6(a), the Tamm SPs are not supported in the $\beta^{\prime} d \ll 1$ region for the air-truncated GMMs investigated in Fig. 4. As it is seen from panels (a) and (b) of Fig. 4, $P L$ values of the Tamm SPs for $d=10 \mathrm{~nm}$ are considerably smaller than those of the SLG, and their localization is not as good as the SPs supported by SLG for a wide range of frequency. This means that when real losses are included, an air-truncated GMM $\left(\varepsilon_{s}<\varepsilon\right)$ supports Tamm SPs that are not as efficient as SPs of a single layer of graphene. By efficient SPs we mean modes with large $P L$ and small $L L$. From these panels, it can be observed that by increasing the period of the metamaterial from $10 \mathrm{~nm}$ to $100 \mathrm{~nm}$, the frequency range in which Tamm SPs are supported can be extended. Moreover, the propagation and localization characteristics of these modes can also be improved. Further investigations show that, as far as supporting Tamm SPs with a large $P L$ and small $L L$ is concerned, an air-truncated GMM can be as effective as a single layer of graphene, when $d=300 \mathrm{~nm}$. It should be noticed that in case we take the thickness of the dielectric layers as $300 \mathrm{~nm}$, the period of the metamaterial still remains subwavelength $(d \ll \lambda)$. To be able to achieve better insight into how Tamm SPs are localized in the metamaterial, $\left|E_{x}\right|$ profiles of these modes for different periods of GMMs at different frequencies are also represented in Fig. 5. For ease of comparison, profiles of some appropriate SPs of a SLG are also shown in black solid curves. In agreement with Fig. 4(b), panels (a)-(d) of Fig. 5 clearly illustrate that for an airtruncated GMM, Tamm SPs are almost less localized than the SPs of a SLG. It should be noticed that the oscillations in the exponential decay in the mode profiles are because of the superposition of the coupled SPs supported by each graphene layer in the metamaterial. This superposing leads to the appearance of highly confined Tamm SPs with $\operatorname{Im}\left(K_{B}\right) \gg \operatorname{Re}\left(K_{B}\right)$. Since our goal is to obtain a miniaturized GMM with the capability of supporting Tamm SPs with better guiding characteristics than SPs of a SLG, for the results presented in Fig. 6 we have replaced air with a semi-infinite material of $\varepsilon_{s}=10\left(\varepsilon_{s}>\varepsilon\right)$, as the cladding medium. Figure 6(a) shows $\omega-\beta^{\prime}$ dispersion of the GMM with $d=10 \mathrm{~nm}$ when the permittivity of the cladding semi-infinite material is chosen as 1 (dashed curve, in agreement with Fig. 4) and 10 (solid curve). Notice that the band structure of the system when $\operatorname{Re}\left(\sigma_{g}\right)=0$ is also plotted in this figure to be able to highlight the position of the Tamm SPs in the $\omega-\beta^{\prime}$ diagram. From this figure, it can be seen that the Tamm modes cannot be supported in $\beta^{\prime} d \ll 1$ region and for this reason, the approximate solutions of Eq. (9a) are not applicable in this case (more investigations show that in the lossy case, in the presence of the cap layer, the Tamm SPs are not supported in the long-wavelength region and hence Eq. (9b) is not also applicable). Consequently, it can be concluded that the GMM does not behave as a hyperbolic metamaterial, but the frequency range in which Tamm SPs are supported starts at $18 \mathrm{THz}$, which is lower than $\varepsilon_{x}=0$ frequency of the graphene hyperbolic metamaterial with $d=10 \mathrm{~nm}$. This point holds for the other values of the period $(d=50 \mathrm{~nm}$ and $100 \mathrm{~nm})$. Moreover, in the case $\varepsilon_{s}=10$, modes can be supported in a considerably wider range of frequencies compared to those of an air-truncated GMM. As it is clearly shown in panels (b) and (c) of Fig. 6, when $d=10 \mathrm{~nm}$ : (i) the Tamm SPs can be supported at $16.7<f(\mathrm{THz})<50$. (ii) $P L$ of the Tamm SPs is considerably increased in comparison to those of a SLG; e.g., at $f=16.7 \mathrm{THz} P L$ is almost 2 orders increased for the corresponding Tamm SP. It is noteworthy that this structure is much more effective than using a 1D PC to increase PL of graphene SPs. ${ }^{40}$ (iii) $L L$ of these modes is either smaller or equal to that of a SLG within the mentioned range of frequency (see blue curves in panels (b) and (c) of Fig. 6). (iv) By
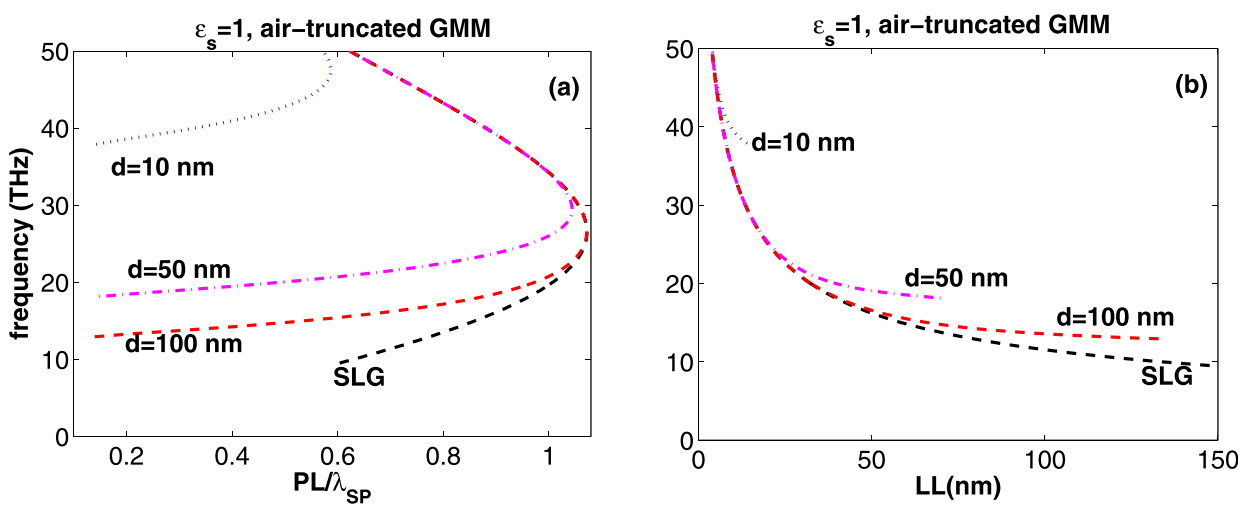

FIG. 4. Normalized propagation length (panel (a)) and localization length (panel (b)) of air-truncated GMMs with different periods are illustrated in these panels. The black-dashed curves in these panels represent the propagations and localization characteristics of a SLG. 

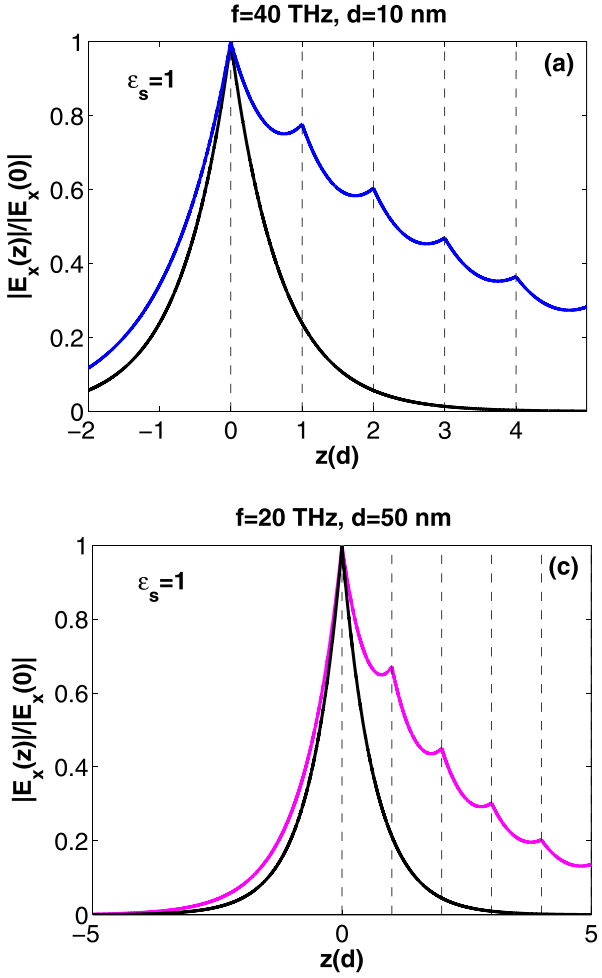
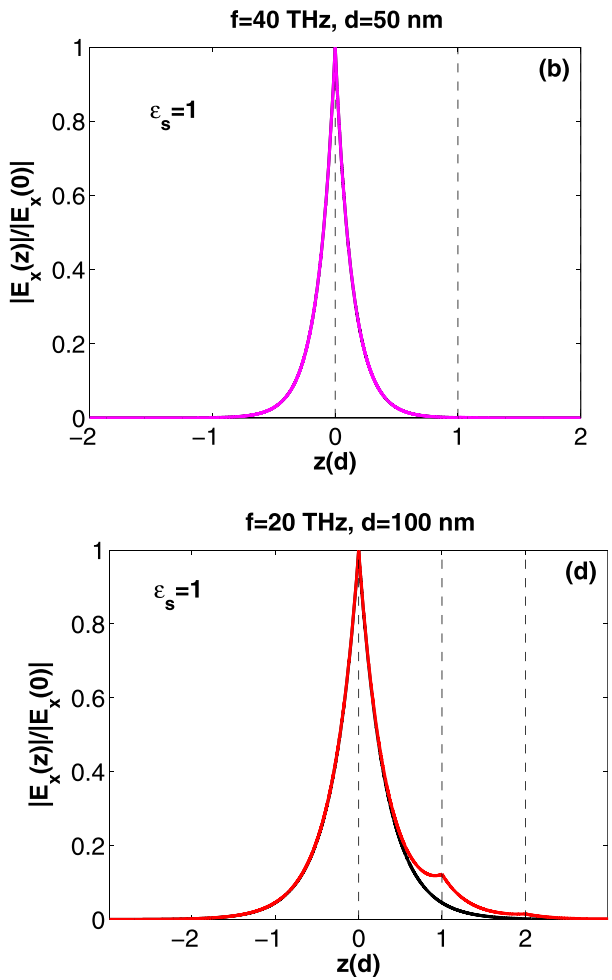

FIG. 5. $\left|E_{x}\right|$ profiles of four typical Tamm surface plasmons supported by air-truncated GMMs $\left(\varepsilon_{s}=1\right)$. Blackdashed vertical lines represent position of the graphene layers in the metamaterial. Black-solid curves show the corresponding mode profiles of SPs of SLG. Notice that in panel (b) mode profiles of Tamm SP and SP of SLG are exactly the same. increasing the period of the metamaterial, it would be also possible to long-range Tamm SPs with a small $L L$ be supported at frequencies lower than $16.7 \mathrm{THz}$ (see pink and red curves in panels (b) and (c)). In support of the results presented in Fig. 6(c), Fig. 7 illustrates the $\left|E_{x}\right|$ profiles of the Tamm SPs of the dielectric-truncated GMMs for different periods and frequencies. As it is seen from this figure, in this case, the decay behavior of the Tamm SPs in the first period of the GMM is much more pronounced than that of the SPs of a SLG (black curves). Here, the Tamm SPs are supported in the bandgap of the metamaterial with $\operatorname{Im}\left(K_{B}\right) \gg \operatorname{Re}\left(K_{B}\right)$. Since the propagation of the modes in the Nth dielectric layer of the GMM can be described by $e^{i(N-1) K_{B} d} H_{y}(z-(N-1) d) e^{i \beta x}$ using Bloch theorem, $\operatorname{Im}\left(K_{B}\right) \gg \operatorname{Re}\left(K_{B}\right)$ conditions for the Tamm SPs enforce considerable decay of these modes in the direction perpendicular to graphene layers. This considerable
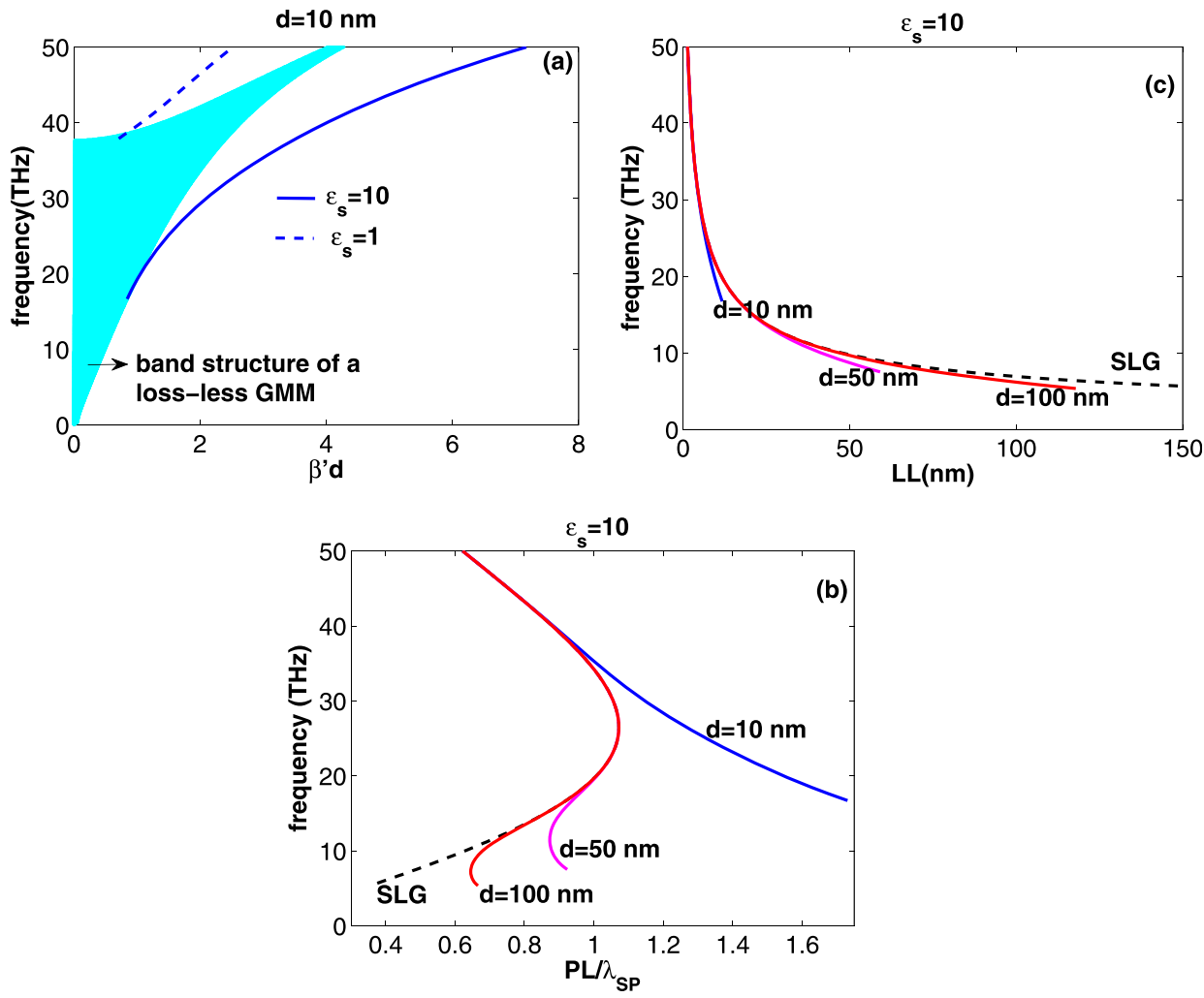

FIG. 6. In panel (a), dashed and solid curves show dispersion of Tamm SPs of air-truncated $\left(\varepsilon_{s}<\varepsilon\right)$ and dielectrictruncated $\left(\varepsilon_{s}>\varepsilon\right) \quad$ GMMs with $d=10 \mathrm{~nm}$. For the ease of locating these dispersions in the $\omega-\beta^{\prime}$ diagram, the band structure of the lossless GMM is also represented in this panel. Panels (b) and (c), respectively, illustrate the normalized $P L$ and $L L$ of the Tamm SPs supported by the GMM for different periods. Black-dashed curves in these panels represent dispersions of SPs of SLG. 

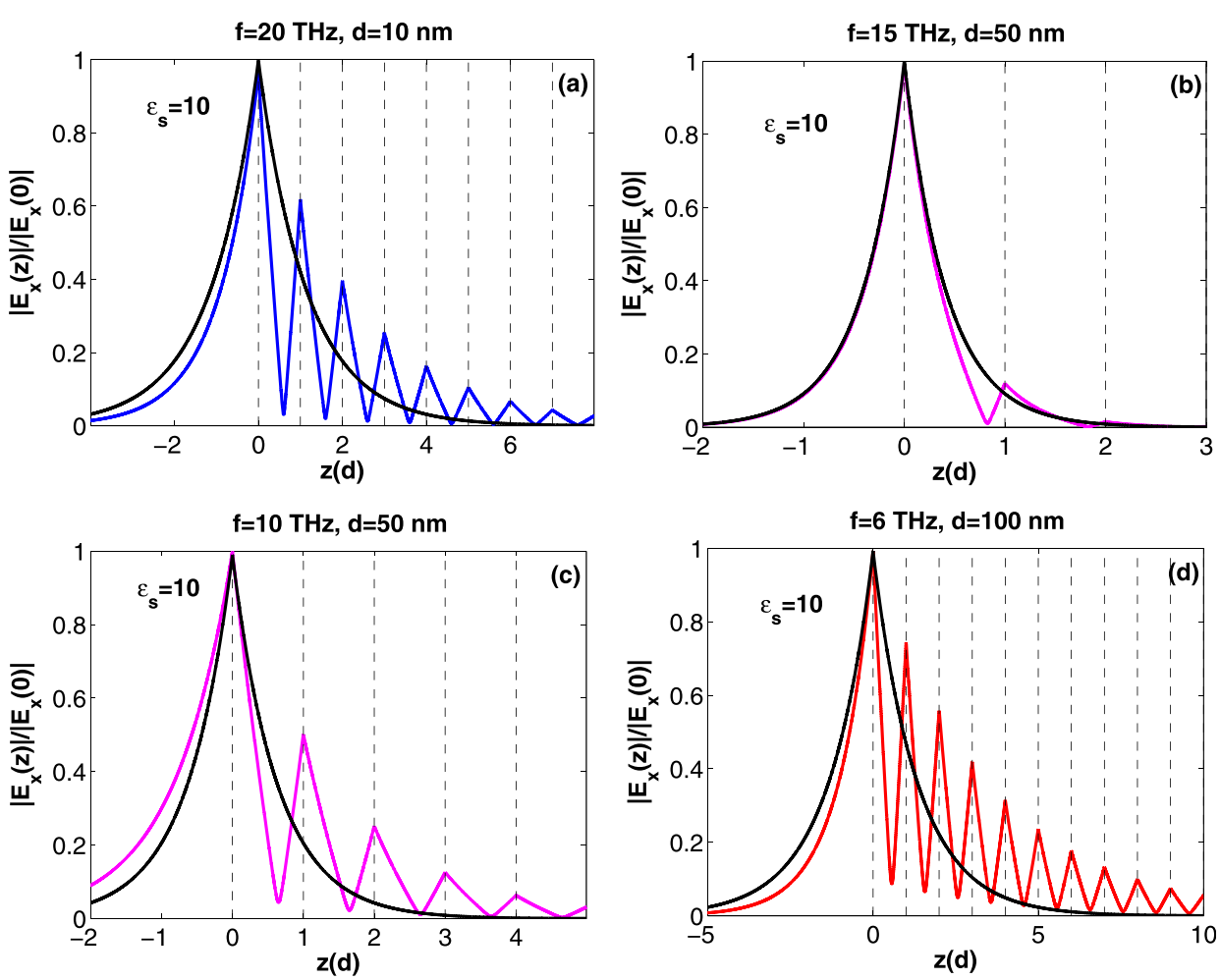

FIG. 7. Similar to Fig. 5, in these panels, black-dashed vertical lines represent the position of the graphene layers in the metamaterial. Black-solid curves show mode profiles of corresponding SPs of SLG. These panels illustrate $\left|E_{x}\right|$ profiles of four typical Tamm SPs supported by dielectric-truncated GMMs $\quad\left(\varepsilon_{s}=10>\varepsilon\right)$ with different periods. The noticeable difference between these plots and those plotted in Fig. 5 is the sharp decay of the Tamm SPs in the first period of the metamaterial. This leads to the higher localization of the modes than that of SPs of SLG, which is in agreement with Fig. 6(c). decay leads to the strong localization of Tamm SPs at the first graphene layer separating the GMM from the truncating medium. Therefore, as it can be seen from the mode profiles illustrated in Fig. 7, for the Tamm SPs the electric field and as a result the electromagnetic energy are mostly concentrated at the first graphene layer instead of distributing within the whole metamaterial. Therefore, using a semi-infinite material with $\varepsilon_{S}>\varepsilon$ as the cladding medium, we are able to considerably increase the propagation length of the Tamm SPs while keeping their localization length as small as possible.

It is noteworthy that for IGI and GIG layered systems where $\mathrm{G}$ and I stand for graphene and insulator, respectively, $1 /|\operatorname{Re}(q)|$ gives us a feasible vision of the energy confinement (or exponential decay length) of the supported SPs. Here, at a typical SP frequency, $1 /|\operatorname{Re}(q)|$ is the length in which the field decays to $1 / e \cong 0.37$ its maximum. For the truncated graphene-insulator metamaterials (GMMs) considered in this manuscript, $1 /|\operatorname{Re}(q)|$, which is obtained by the direct-matching procedure, defines the exponential decay length of the Tamm SPs into the first period of the metamaterial adjacent to the truncating medium. To make this point clear, we compare the value of $L L$ at $f=20 \mathrm{THz}$ for the blue curve presented in Fig. 6(c) with the corresponding decay length of the mode profile illustrated in the first period of panel (a) of Fig. 7. According to Fig. 6(c), the $L L$ of the Tamm SPs supported by the dielectric-truncated GMM with $d=10 \mathrm{~nm}$ is $9.5 \mathrm{~nm}$, and that of SPs of a single layer graphene bounded with the corresponding dielectric media is $11.5 \mathrm{~nm}$. These numbers are in exact agreement with the corresponding spatial modal size presented in Fig. 7(a) when the mode profiles reach $1 / e$ their maximum. Another important point is that, as can be seen from Fig. 7, at $z=1 d, 2 d$, $3 d, \ldots$ the Tamm modes show larger field amplitudes than those of a single layer of graphene, while the values of $L L$ illustrated in Fig. 6(c) for the Tamm SPs represent smaller values as compared to those of a SLG. As explained above, the reason for this difference is that $1 /|\operatorname{Re}(q)|$ defines the exponential decay length of the Tamm SPs into the first period of the metamaterial adjacent to the truncating medium not into the whole medium. It should be noticed that our goal in this paper is to design optimal structures to support Tamm SPs with large PL and small $L L$, in comparison with those of a single layer of graphene. Therefore, to this aim, the method used in this paper for the investigation of the localization length of the modes, which is based on obtaining the modal confinement in the first period of the metamaterial, gives us a fair practical estimate of the spatial modal size of the Tamm surface plasmons. Moreover, a practical point should be highlighted here is that, according to the mode profiles, 10 layers of graphene would be sufficient in order for a multilayer graphene system to act as a GMM with expected optical responses.

From a practical point of view, having a semi-infinite material with $\varepsilon_{s}=10$ as the cladding medium of the metamaterial may be unclear. Hence, our final aim in this paper is to modify this geometry to a feasible design. Considering a thick cap layer of width $d_{c}=5 d$ and $\varepsilon_{c}=10$ in between the GMM and air $\left(\varepsilon_{s}=1\right)$, the propagation and localization characteristics of the Tamm SPs are represented in Fig. 8. By comparing panels (a) and (b) of the above figure with panels (b) and (c) of Fig. 6, it is straightforward to conclude that by using a thick cap layer of width $d_{c}=5 d$ and $\varepsilon_{c}=10$ in the graphene metamaterial and choosing an appropriate period, referred to as optimal design, it would be possible for the system to support long-range Tamm SPs within 5.5-50 THz range of frequency. 


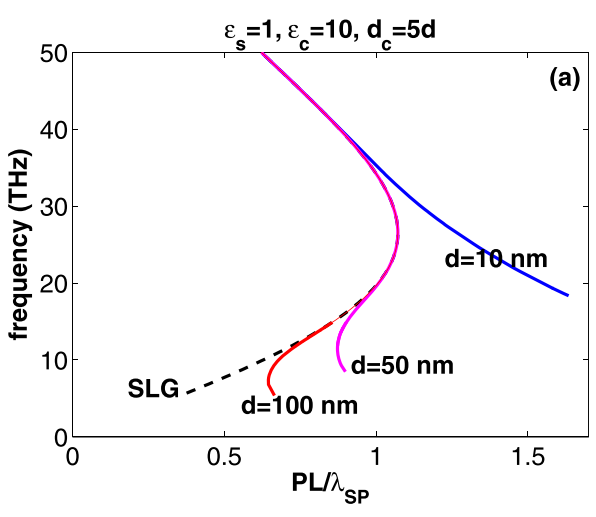

\section{CONCLUSION}

In conclusion, we have theoretically examined the propagation and localization characteristics of low-loss Tamm surface plasmons supported by multilayer graphenedielectric metamaterials. We have demonstrated that by taking the subwavelength thickness of the dielectric layers as $300 \mathrm{~nm}$, Tamm SPs with moderate propagation and localization lengths, comparable with those of a single layer of graphene, are supported by an air-truncated graphene metamaterial. On the other hand, if the miniaturized graphene structure with $10 \mathrm{~nm}<d<100 \mathrm{~nm}$ is truncated by a thick dielectric cap layer of $\varepsilon_{c a p}>\varepsilon_{\text {dielectric }}$, highly localized long-range Tamm surface plasmons are supported by the system in a wide range of frequency extending from $\mathrm{THz}$ to mid-IR. In fact, in this case, it is possible to increase the PL of the highly confined modes up to two orders at some frequencies, as compared with a single layer of graphene. These realistically optimal graphene-dielectric metamaterial structures, which do not act as hyperbolic metamaterials while supporting Tamm surface plasmons, are appropriate for feasibly designing graphene metamaterials, which are beneficial to guiding and sensing applications.

\section{ACKNOWLEDGMENTS}

This work was supported by the Project Nos. DPTHAMIT, NATO-SET-193, TUBITAK-113E331, and TUBITAK-114E374. The authors (E.O. and H.C.) also acknowledge partial support from the Turkish Academy of Sciences.

${ }^{1}$ I. Tamm, Zh. Eksp. Teor. Fiz. 3, 34 (1933).

${ }^{2}$ P. Yeh, A. Yariv, and C.-S. Hong, J. Opt. Soc. Am. 67, 423 (1977).

${ }^{3}$ M. Kaliteevski, I. Iorsh, S. Brand, R. A. Abram, J. M. Chamberlain, A. V. Kavokin, and I. A. Shelykh, Phys. Rev. B 76, 165415 (2007).

${ }^{4}$ J. A. Polo, Jr. and A. Lakhtakia, Laser Photonics Rev. 5, 234 (2011).

${ }^{5}$ A. I. Rahachou and I. V. Zozoulenko, J. Opt. Soc. Am. B 23, 1679 (2006).

${ }^{6}$ V. N. Konopsky and E. V. Alieva, Anal. Chem. 79, 4729 (2007).

${ }^{7}$ A. V. Kavokin, I. A. Shelykh, and G. Malpuech, Phys. Rev. B 72, 233102 (2005).

${ }^{8}$ A. Kavokin, I. Shelykh, and G. Malpuech, Appl. Phys. Lett. 87, 261105 (2005).

${ }^{9}$ S. A. Maier, Plasmonics: Fundamentals and Applications (Springer, Berlin, 2007).

${ }^{10}$ H. Hajian, A. SoltaniVala, and M. Kalafi, Physica B: Condens. Matter 406, 4094 (2011).
FIG. 8. Panels (a) and (b), respectively, represent the normalized propagation length and localization length of Tamm SPs supported by cap-truncated GMMs for different periods, $d$. The thickness of the cap layer is taken as $d_{c}=5 d$. Similar to the previous figures, dashed-black curves show the dispersion of modes of SLG.

${ }^{11}$ J. D. Joannopoulos, S. G. Johnson, J. N. Winn, and R. D. Meade, Photonic Crystals: Molding the Flow of Light, 2nd ed. (Princeton University Press, Princeton, NJ, 2008).

${ }^{12}$ H. Hajian, A. Soltani-Valaa, and M. Kalafi, Opt. Commun. 283, 4847 (2010).

${ }^{13}$ A. P. Vinogradov, A. V. Dorofeenko, A. M. Merzlikin, and A. A. Lisyansky, Phys.-Usp. 53, 243 (2010).

${ }^{14}$ A. Namdar, I. V. Shadrivov, and Yu. S. Kivshar, Appl. Phys. Lett. 89, 114104 (2006).

${ }^{15}$ I. Iorsh, A. Orlov, P. A. Belov, and Yu. S. Kivshar, Appl. Phys. Lett. 99, 151914 (2011).

${ }^{16}$ I. Iorsh, P. A. Belov, A. A. Zharov, I. V. Shadrivov, and Y. S. Kivshar, Phys. Rev. A 86, 023819 (2012).

${ }^{17}$ C. L. Cortes, W. Newman, S. Molesky, and Z. Jacob, J. Opt. 14, 063001 (2012).

${ }^{18}$ A. Poddubny, I. Iorsh, P. Belov, and Y. Kivshar, Nat. Photonics 7, 948 (2013).

${ }^{19}$ L. Ferraria, C. Wub, D. Lepaged, X. Zhang, and Z. Liu, Prog. Quantum Electron. 40, 1 (2015).

${ }^{20}$ A. K. Geim and K. S. Novoselov, Nat. Mater. 6, 183 (2007).

${ }^{21}$ F. Bonaccorso, Z. Sun, T. Hasan, and A. C. Ferrari, Nat. Photonics 4, 611 (2010).

${ }^{22}$ A. N. Grigorenko, M. Polini, and K. S. Novoselov, Nat. Photonics 6, 749 (2012).

${ }^{23}$ A. Vakil and N. Engheta, Science 332(6035), 1291 (2011).

${ }^{24}$ L. A. Falkovsky, J. Phys.: Conf. Ser. 129, 012004 (2008).

${ }^{25}$ I. Iorsh, I. S. Mukhin, I. V. Shadrivov, P. A. Belov, and Y. S. Kivshar, Phys. Rev. B 87, 075416 (2013).

${ }^{26}$ M. A. K. Othman, C. Guclu, and F. Capolino, Opt. Exp. 21, 7614 (2013).

${ }^{27}$ Y. C. Chang, C. H. Liu, C. H. Liu, S. Zhang, S. R. Marder, E. E. Narimanov, Z. Zhong, and T. B. Norris, Nat. Commun. 7, 10568 (2016).

${ }^{28}$ D. A. Smirnova, I. Iorsh, I. V. Shadrivov, and Y. S. Kivshar, JETP Lett. 99, 456 (2014).

${ }^{29}$ B. Zhu, G. Ren, S. Zheng, Z. Lin, and S. Jian, Opt. Exp. 21, 17089 (2013).

${ }^{30}$ T. Zhang, L. Chen, and X. Li, Opt. Exp. 21, 20888 (2013).

${ }^{31}$ K. V. Sreekanth, A. De Luca, and G. Strangi, Appl. Phys. Lett. 103, 023107 (2013).

${ }^{32}$ I. S. Nefedov, C. A. Valaginnopoulos, and L. A. Melnikov, J. Opt. 15, 114003 (2013).

${ }^{33}$ R. Chern, D. Han, Z. Q. Zhang, and C. T. Chan, Opt. Exp. 22, 31677 (2014).

${ }^{34}$ D. Smirnova, P. Buslaev, I. Iorsh, I. V. Shadrivov, P. A. Belov, and Y. S. Kivshar, Phys. Rev. B 89, 245414 (2014).

${ }^{35}$ Y. Xiang, J. Guo, X. Dai, S. Wen, and D. Tang, Opt. Exp. 22, 3054 (2014).

${ }^{36}$ S. V. Zhukovsky, A. Andryieuski, J. E. Sipe, and A. V. Lavrinenko, Phys. Rev. B 90, 155429 (2014).

${ }^{37}$ H. Hajian, A. Soltani-Vala, and M. Kalafi, Opt. Commun. 292, 149 (2013).

${ }^{38}$ Yu. V. Bludov, N. M. R. Peres, and M. I. Vasilevskiy, J. Opt. 15, 114004 (2013).

${ }^{39}$ M. Steeslicka, R. Kucharczyk, A. Akjouj, B. Djafari-Rouhani, L. Dobrzynski, and S. G. Davison, Surf. Sci. Rep. 47, 93 (2002).

${ }^{40}$ H. Hajian, A. Soltani-Vala, and M. Kalafi, J. Appl. Phys. 114, 033102 (2013).

${ }^{41}$ S. V. Zhukovsky, O. Kidwai, and J. E. Sipe, Opt. Express 21, 14982 (2013). 